\title{
2017 Year End Experiment Planning and Management Summary Report
}

September 2017

Katie Anderson

Nick Meacham

Debra Utterbeck

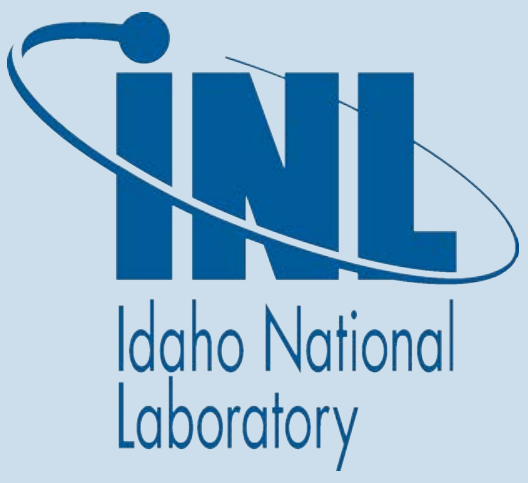

\author{
Idaho National Laboratory \\ Nuclear Science User Facilities \\ Idaho Falls, Idaho 83415
}

The INL is a U.S. Department of Energy National Laboratory operated by Battelle Energy Alliance. 


\section{CONTENTS}

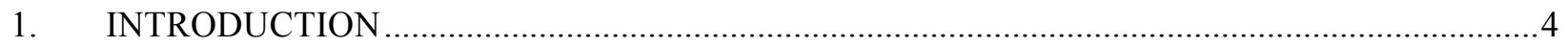

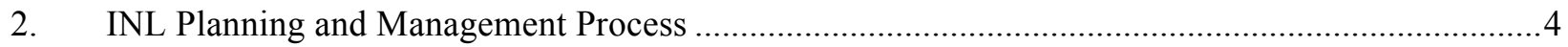

3. NSUF EXPERIMENT IRRADIATIONS IN THE ADVANCED TEST REACTOR .......................5

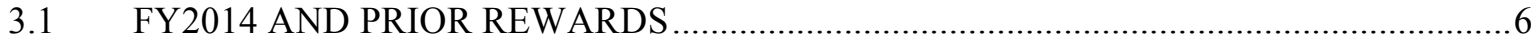

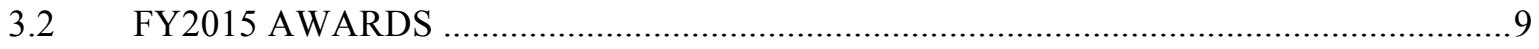

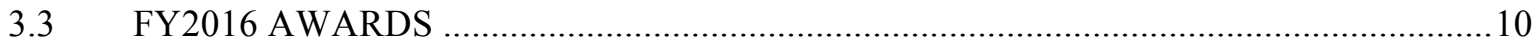

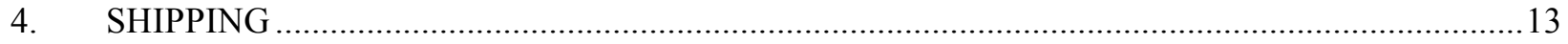

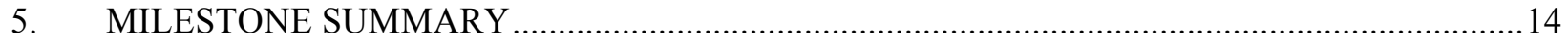

\section{FIGURES}

Figure 1. Overall process flow for executing nuclear material experiments........................4

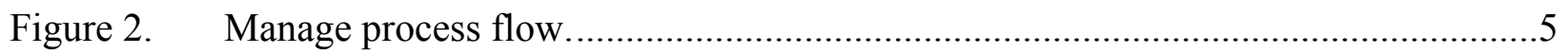

Figure 3. ATR Cross Section Highlighting NSUF Identified Irradiation Locations................6

Figure 4. BSU-269 Rodlet and Capsule Assembly Schematic ........................................

Figure 5. BSU-269 Irradiation Facility with Baskets Inserted .......................................

Figure 6. UCF capsule with TEM discs, foil specimens, and spacers ...............................

Figure 7. BSU-8242 Capsule and Fixture Layout .....................................................

Figure 8. CSM-10584 Capsule and Fixture Layout.................................................... 10

Figure 9. N-SERT Capsule and Fixture Layout .........................................................11

Figure 10. Schematic of GEH-10393 Capsule and Specimens ..........................................12

\section{TABLES}

Table 1. Milestone Summary .14 


\section{INTRODUCTION}

The Nuclear Science User Facilities (NSUF) are part of a growing number of Department of Energy user facilities in the U.S., and is the only national designated nuclear energy user facility. Through a peerreviewed proposal process, NSUF provides external research teams cost-free access to reactor, postirradiation examination (PIE) and beamline capabilities at Idaho National Laboratory (INL) and a diverse mix of affiliated partner institutions at universities, national laboratories and industry facilities located across the country.

In Fiscal Year 2017 (FY17), the NSUF sponsored planning and management activities at INL of two ongoing irradiation experiments and design, analysis, and reactor insertion of six new Advanced Test Reactor (ATR) experiment irradiations. Additionally, shipping hardware was fabricated and shipments of specimens to and from the INL were made. Experiment managers (EMs) and the shipping coordinator were key participants in the ATR Integrated Strategic Operating Plan (ISOP) development, baseline work planning, variance reporting, detailed work planning, and cost estimating of Fiscal Year 2018 (FY18) proposal for consideration during the FY18 call. General support for instrumentation hardware, data analysis via software licenses (ABAQUS), vendor qualification, as-run analysis, Safety Analysis Report revisions, and activities supporting experimental methods were performed.

\section{INL Planning and Management Process}

A major focus of INL's mission is the experimental testing of advanced and current-generation nuclear fuels and materials. Scientific experiments provide deeper understanding of basic radiation damage processes that can determine the basis for performance improvements and verification of modeling assumptions. Because these data and subsequent analyses are among the INL's primary products, sound execution of this research is essential. The INL follows a defined sequence of activities and standard practices that will ensure efficient execution of experiments and yield high quality data (see Figure 1 and Appendix A).

\section{Execute Nuclear Materials Experiment}

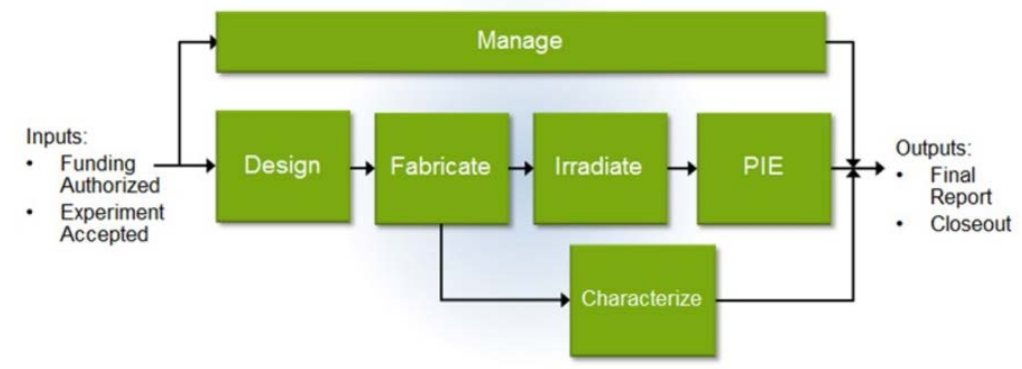

Figure 1. Overall process flow for executing nuclear material experiments.

The manage phase spans the life-cycle of the experiment (see Figure 2). This phase begins once the experiment has been accepted and funding secured and ends when the final experiment report is issued and the experiment has been closed out. Execution of the experiment life cycle is typically segregated and managed between an Irradiation EM and a PIE EM. 


\section{Manage (Level 3)}

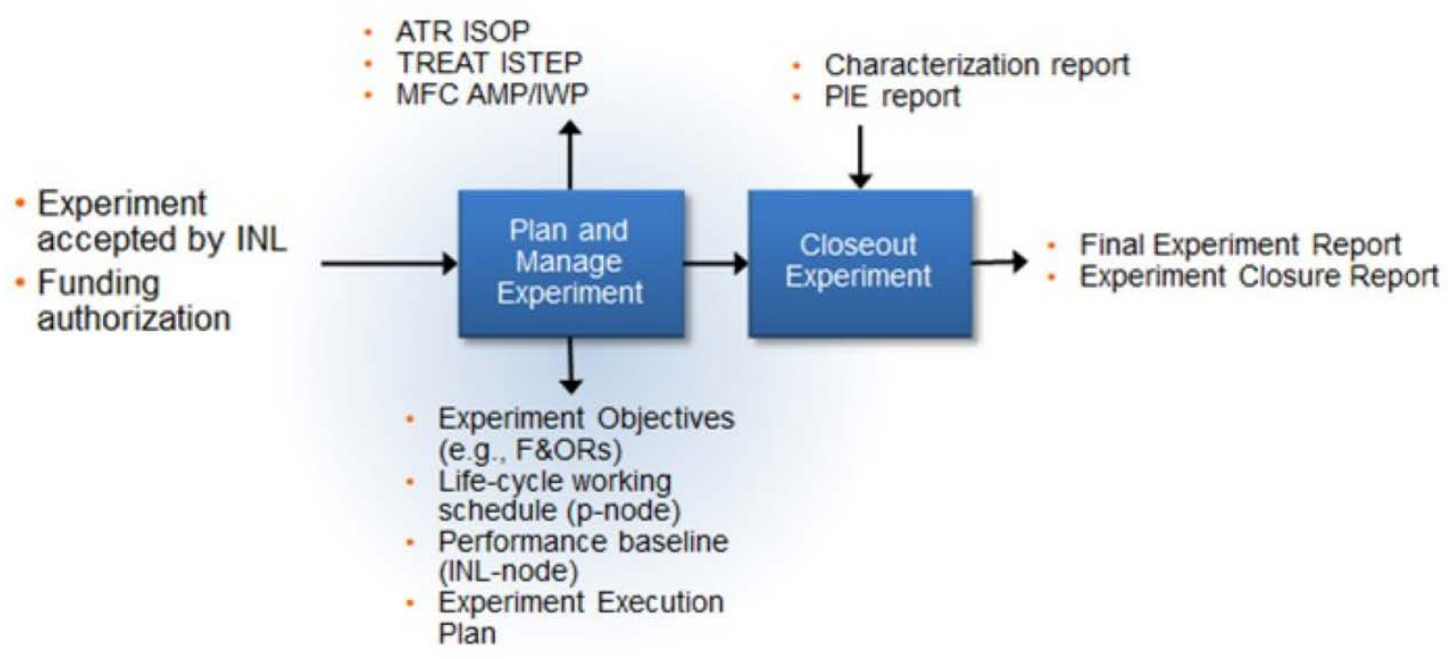

Figure 2. Manage process flow.

Based on the program's overall objectives established in the accepted proposal, the Program Technical Lead, with support from the core experiment team, planned the life-cycle of the experiment. The Experiment Execution Plan was developed and contains the experiment objectives, life-cycle schedule and budget, applicable requirements, key deliverables and experiment-specific schedules.

\section{NSUF EXPERIMENT IRRADIATIONS IN THE ADVANCED TEST REACTOR}

The ATR is a water-cooled, high-flux test reactor, with a unique serpentine design that allows large power variations among its flux traps. The reactor's curved fuel arrangement places fuel closer on all sides of the flux trap positions than is possible in a rectangular grid. The reactor has nine high neutron flux traps and 68 additional irradiation positions inside the reactor core reflector tank. Experiment positions vary in size from $0.5 "$ to $5.0^{\prime \prime}$ in diameter and all are 48 " long. The peak thermal flux is $1 \times 10^{15} \mathrm{n} / \mathrm{cm}^{2}$-sec and fast flux is $5 \times 10^{14} \mathrm{n} / \mathrm{cm}^{2}$-sec when operating at full power of $250 \mathrm{MW}$. The irradiation environment for the NSUF test assemblies (capsules and baskets) is the ATR primary coolant system. ATR primary coolant system is clean water sampled three times per day and maintained at the slightly acidic $\mathrm{pH}$ of $5.0-5.3$. Chlorides are controlled to $<0.1 \mathrm{ppm}$ (normal $<0.05 \mathrm{ppm}$ ) and normal gross beta-gamma activity is $<$ $0.16 \mu \mathrm{Ci} / \mathrm{ml}$. Control of $\mathrm{pH}$, solids, and chlorides are maintained using ion exchange columns, filters, and chemistry control additives within the system boundaries.

Figure 3 below shows a cross-sectional view of the ATR highlighting the NSUF's 11 positions identified for irradiation of experiments. 


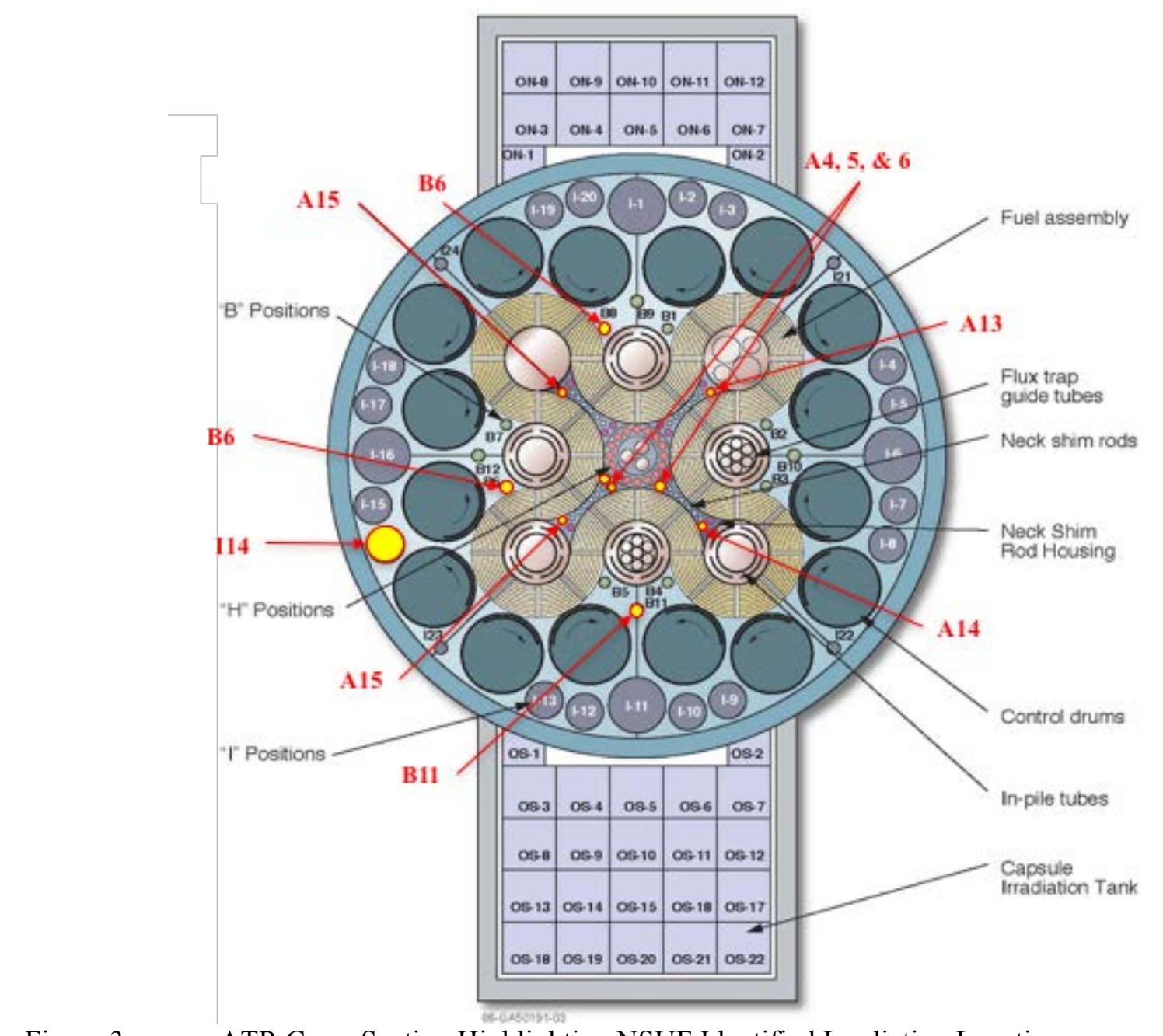

Figure 3. ATR Cross Section Highlighting NSUF Identified Irradiation Locations.

The following sections provide a summary of activities performed in FY17 in support of the NSUF Program's experiment irradiations.

\subsection{FY2014 AND PRIOR REWARDS}

\section{Boise State University 269 (BSU-269)}

The objective of the BSU-269 experiment is to investigate the fuel clad chemical interaction behavior of $\mathrm{U}_{3} \mathrm{Si}_{2}$ and various candidate cladding materials to multiple burnups. The experiment was designed as a drop-in static capsule being irradiated in the ATR I14 position located in the southwest lobe. Irradiation is planned for up to 16 ATR cycles, reaching burnups of $\sim 10 \mathrm{MW} \mathrm{d} / \mathrm{kgU}$ and $20 \mathrm{MW} \mathrm{d} / \mathrm{kgU}$.

During FY17, final design and analysis of the experiment were completed in the first quarter and fuel pellets and experiment component fabrication was completed by the end of June. The experiment was assembled and all documentation was completed in July, identifying the experiment as ready for reactor 
insertion. Figure 4 below shows a schematic of the rodlet and capsules assembly and Figure 5 is a photo of the BSU-269 experiment irradiation facility with three baskets inserted and ready for reactor insertion.

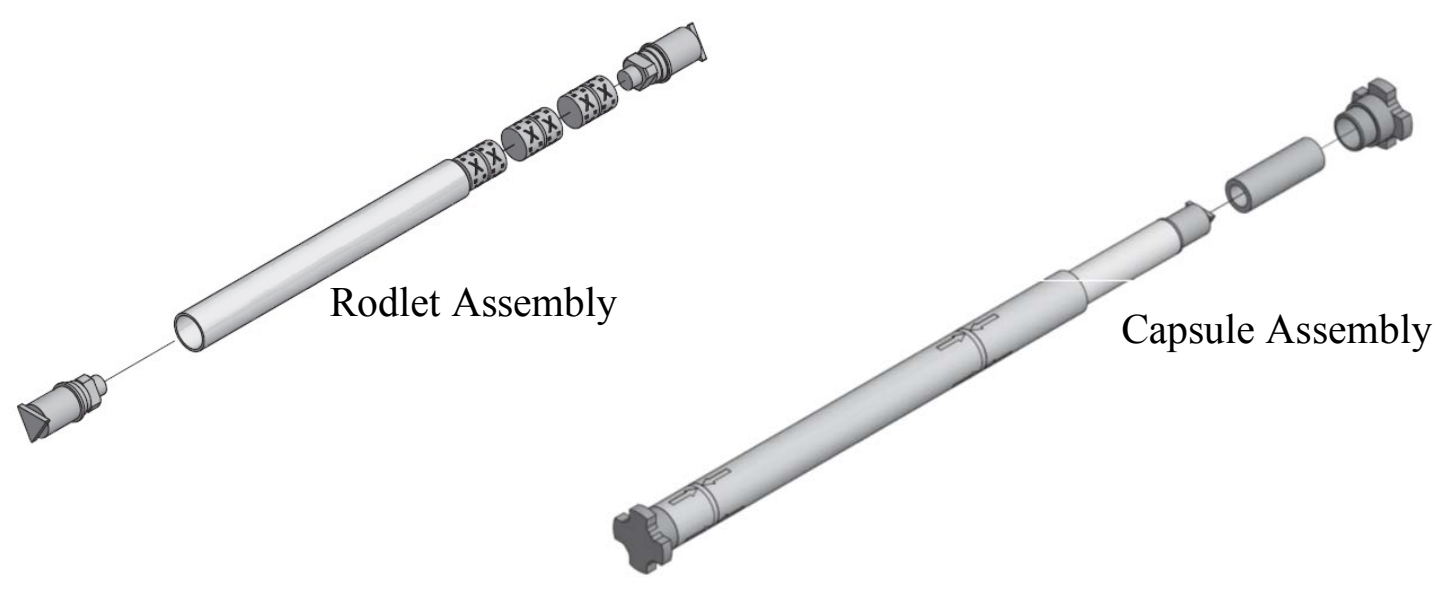

Figure 4. BSU-269 Rodlet and Capsule Assembly Schematic

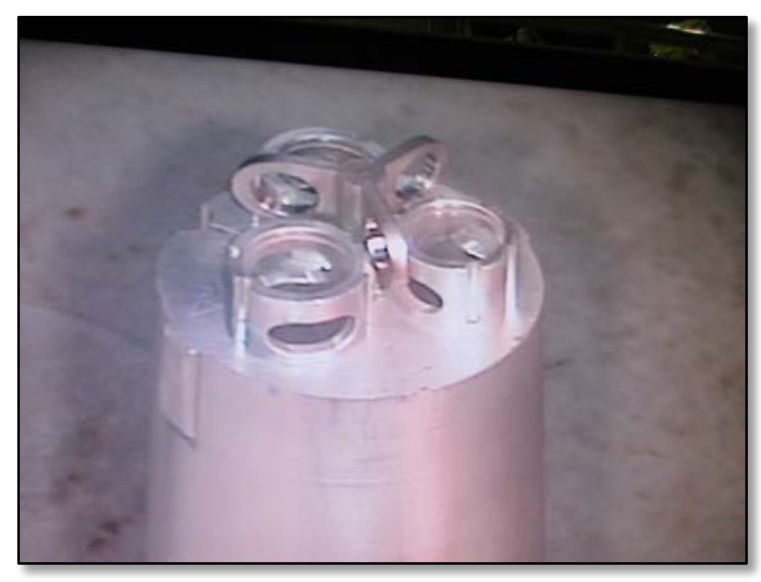

Figure 5. BSU-269 Irradiation Facility with Baskets Inserted

The BSU-269 experiment is scheduled to be irradiated during ATR cycle 162A with startup scheduled for early October 2017.

\section{University of Central Florida 242 (UCF-3)}

UCF-3 is the third stage of the UCF experiments and is designed as a drop-in static capsule. The experiment consists of 13 individual capsule assemblies (see Figure 6) placed in a stainless steel basket. The goal of the UCF-3 experiment is to irradiate U-Zr and U-Mo based metallic specimens at a fluence of $1 \mathrm{dpa}$ and at temperatures ranging from $150^{\circ} \mathrm{C}$ to $800^{\circ} \mathrm{C}$. The specimens consist of a variety of materials including depleted uranium (DU), depleted uranium with 10 weight percent molybdenum (DU $\left.{ }^{10} \mathrm{Mo}\right)$, aluminum $(\mathrm{Al})$, zirconium $(\mathrm{Zr})$, iron $(\mathrm{Fe})$, and iron alloyed with 15 weight percent chromium $\left(\mathrm{Fe}^{15} \mathrm{Cr}\right)$. 
Temperature monitors and flux monitors were included in the capsule assemblies to validate irradiation results.
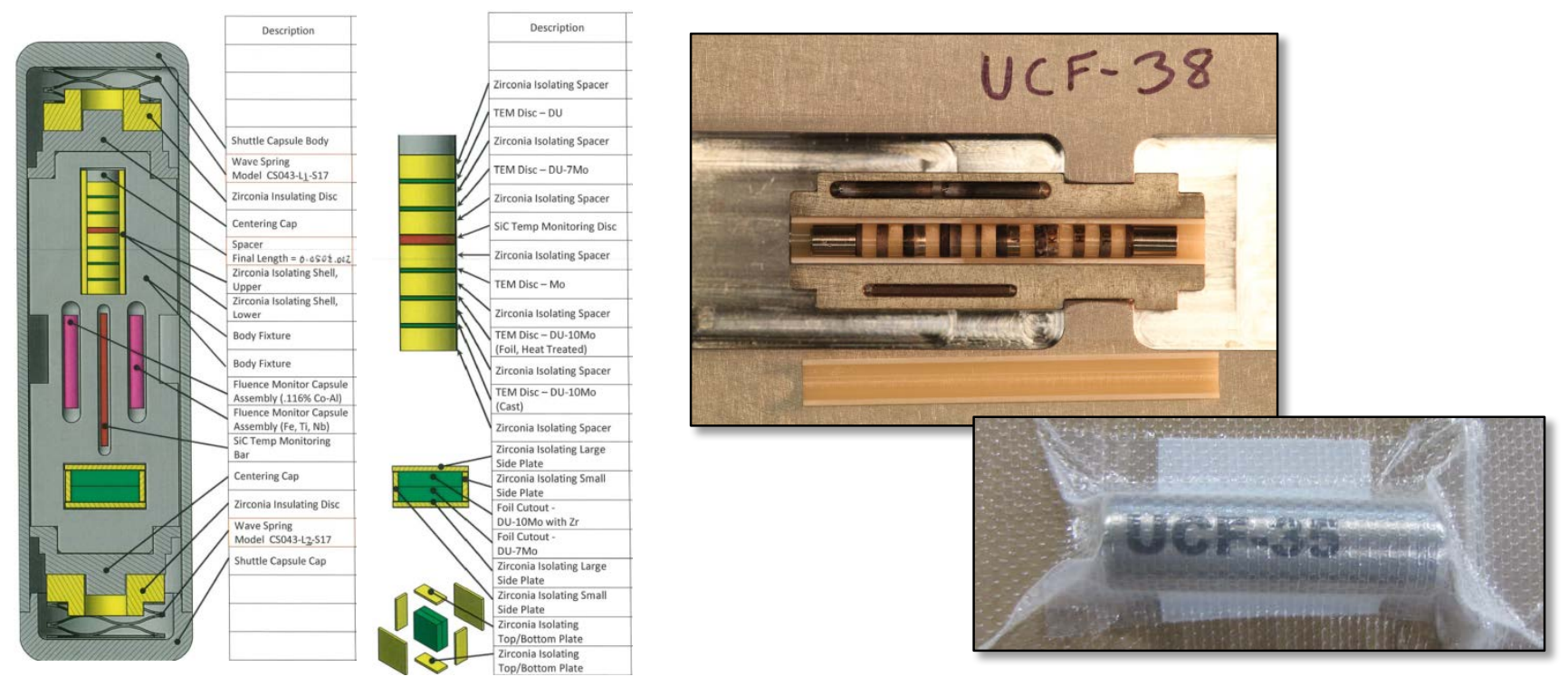

Figure 6. UCF capsule with TEM discs, foil specimens, and spacers

UCF-3 component and specimen fabrication and final assembly was completed by the first quarter of FY17 and the experiment was inserted into ATR's B8 position in December 2016. The experiment will remain in the ATR for four irradiation cycles with completion planned for late in the third quarter or early fourth quarter of FY18, contingent on ATR operation. The second stage UCF-2 experiment is currently on hold due to the ATR hydraulic shuttle irradiation system (HSIS) being out of service. Re-installation of the HSIS system during Core Internal Change Out planned to begin in February 2020, is being evaluated. UCF-2 test specimens and hardware are being stored pending a viable irradiation path forward.

\section{EPRI-Zirconium Growth (EPRI-ZG)}

The EPRI-ZG experiments are designed as drop-in static capsules and are being performed to examine the irradiation growth rate of several zirconium alloys as a function of fluence and hydrogen content and to evaluate the post-irradiation microstructure using TEM to evaluate the growth mechanism.

The EPRI-ZG-C and EPRI-ZG-D experiments are continuing their scheduled irradiation cycles until they reach their cumulative programmatic dpa targets. Achieving these targets is expected to take several more cycles of irradiation. Current projections estimate that EPRI-ZG-C has at least seven cycles remaining to reach its target of $20 \mathrm{dpa}$ and EPRI-ZG-D has at least 16 cycles remaining before reaching its target of 30 dpa. The experiments are being irradiated in outboard A positions whenever reactor cycle lobe powers are within $\pm 1 \mathrm{MW}$ of the nominal power values for which the experiments were designed. 


\subsection{FY2015 AWARDS}

\section{Boise State University 8242}

The BSU-8242 is a drop-in static materials experiment that consists of seven capsules planned for irradiation in three ATR inboard A positions beginning in ATR cycle 164A. The objective of this experiment is to assess the viability of using alloys manufactured by powder metallurgy and hot isostatic pressing (PM-HIP) and to compare the irradiation effects of PM-HIP alloys to alloys fabricated via other, more conventional processes. The capsules and fixtures are made from aluminum and contain specimens, melt wires, fluence monitors, and $\mathrm{SiC}$ temperature monitors (see Figure 7).

The experiment will be irradiated for a total of four ATR cycles. The conceptual design review was completed in December 2016 and final design review was completed August 2017. The Experiment Safety Assurance (ESA) package has been drafted and is currently being reviewed by the Safety Operations Review Committee (SORC). Fabrication of the material specimens is nearing completion and fabrication of the experiment hardware has begun and is expected to be complete by the first quarter of 2017 with experiment assembly to follow.

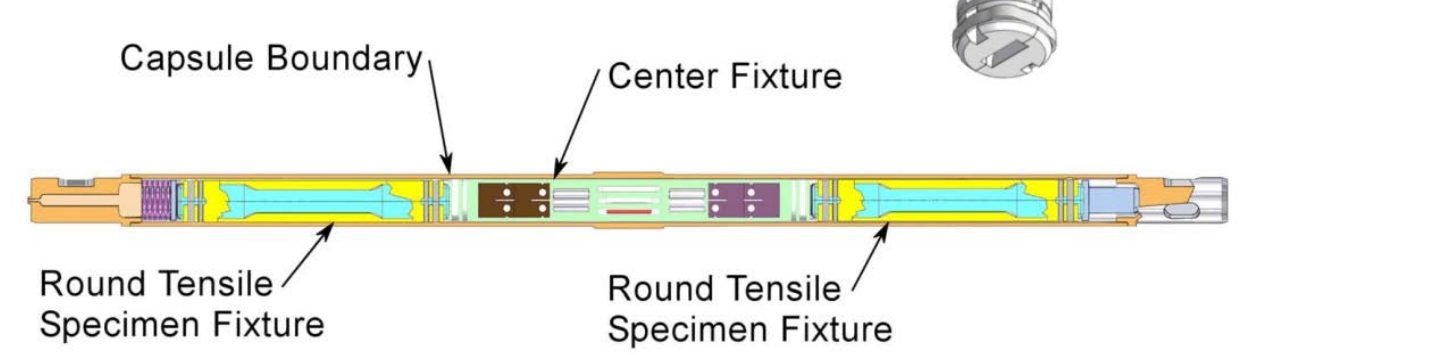

Figure 7. BSU-8242 Capsule and Fixture Layout 


\subsection{FY2016 AWARDS}

\section{Colorado School of Mines 10584}

CSM-10584 is a drop-in static capsule materials experiment whose objective is to collect first-ever irradiation performance data for stainless steel and Inconel specimens produced using commercially available additive manufacturing (3D printing) techniques.

The experiment consists of five capsules to be irradiated for two cycles in ATR's B5 position beginning with cycle 164A. The capsules are stacked vertically end-to-end and the test train consists of two low, two medium, and one high dpa capsules, within which the specimens are held in holders. The two low dpa capsules on each end of the experiment test train will be removed after one cycle of irradiation. The low dpa capsules will be replaced with spacers and the experiment will be re-inserted into the reactor for a second cycle to achieve the desired dpa for the medium and high dpa capsules. A total of 288 specimens (96 miniature tensile specimens, 96 TEM discs, and 96 thermophysical proptery discs) will be dispersed among the five capsules. Figure 8 show a schematic of the fixtures and the fixtures inside the capsule.
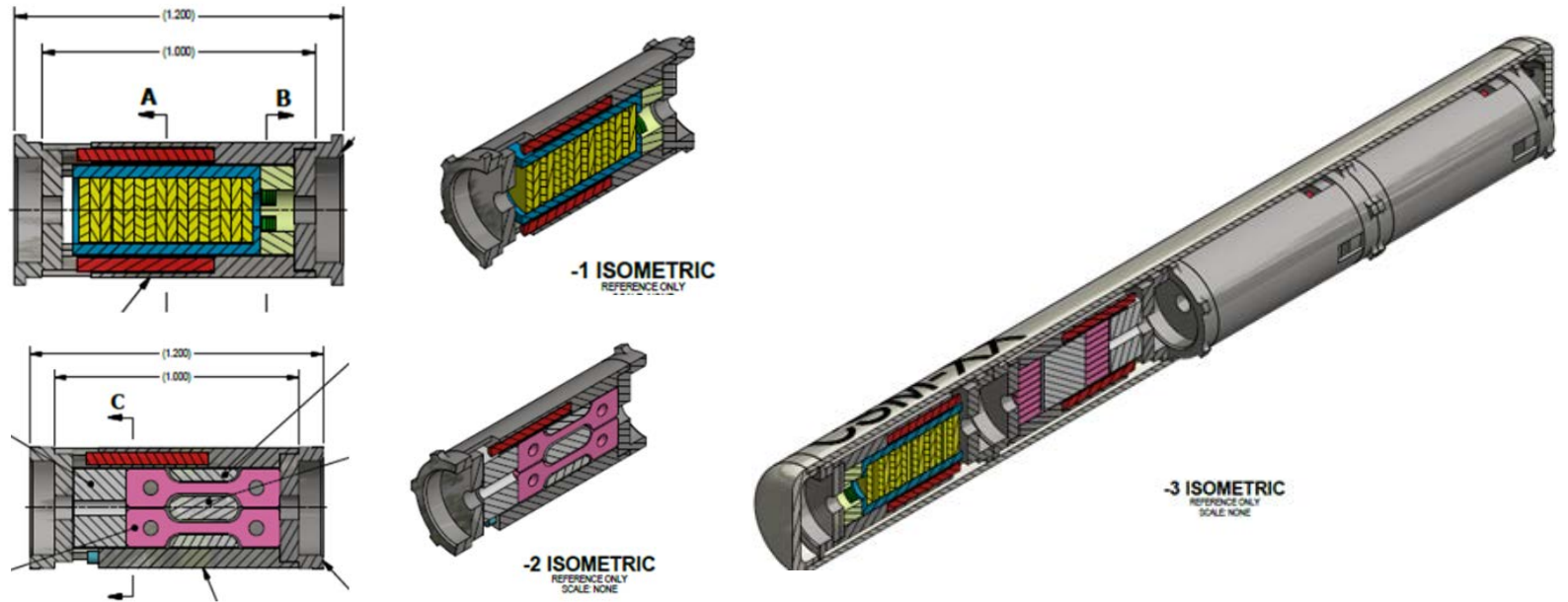

Figure 8. $\quad$ CSM-10584 Capsule and Fixture Layout

The conceptual design review was completed in January 2017 and the final design review was completed and on June 30, 2017, meeting the June 30, 2017 "Final Design Complete" milestone. In addition, the ESA is complete and undergoing review by SORC. The fabrication of the material specimens is underway and specimens are expected to be received by the end of October 2017. The experiment hardware is being fabricated at INL and is planned to finish by October 2017, followed by experiment assembly upon receipt of the specimens. 


\section{Idaho State University 10537 (N-SERT)}

The ISU-10537 experiment (also known as N-SERT) is a drop-in static capsule materials experiment consisting of four capsules to be irradiated in the ATR's B6 position beginning with cycle 164A. The purpose of this experiment is to establish the irradiation performance of ultrafine-grained and nanocrystalline variants of reactor structural and cladding steels produced by severe plastic deformation manufacturing techniques. In addition, high entropy alloy materials will be irradiated. Twelve different materials consisting of 23 sample sets representing various grain sizes-coarse, ultrafine, nanocrystalline, and amorphous will be irradiated. Each capsule (see

Figure 9) will contain 46 TEM specimens, 24 hardness specimens and 56 tensile specimens.

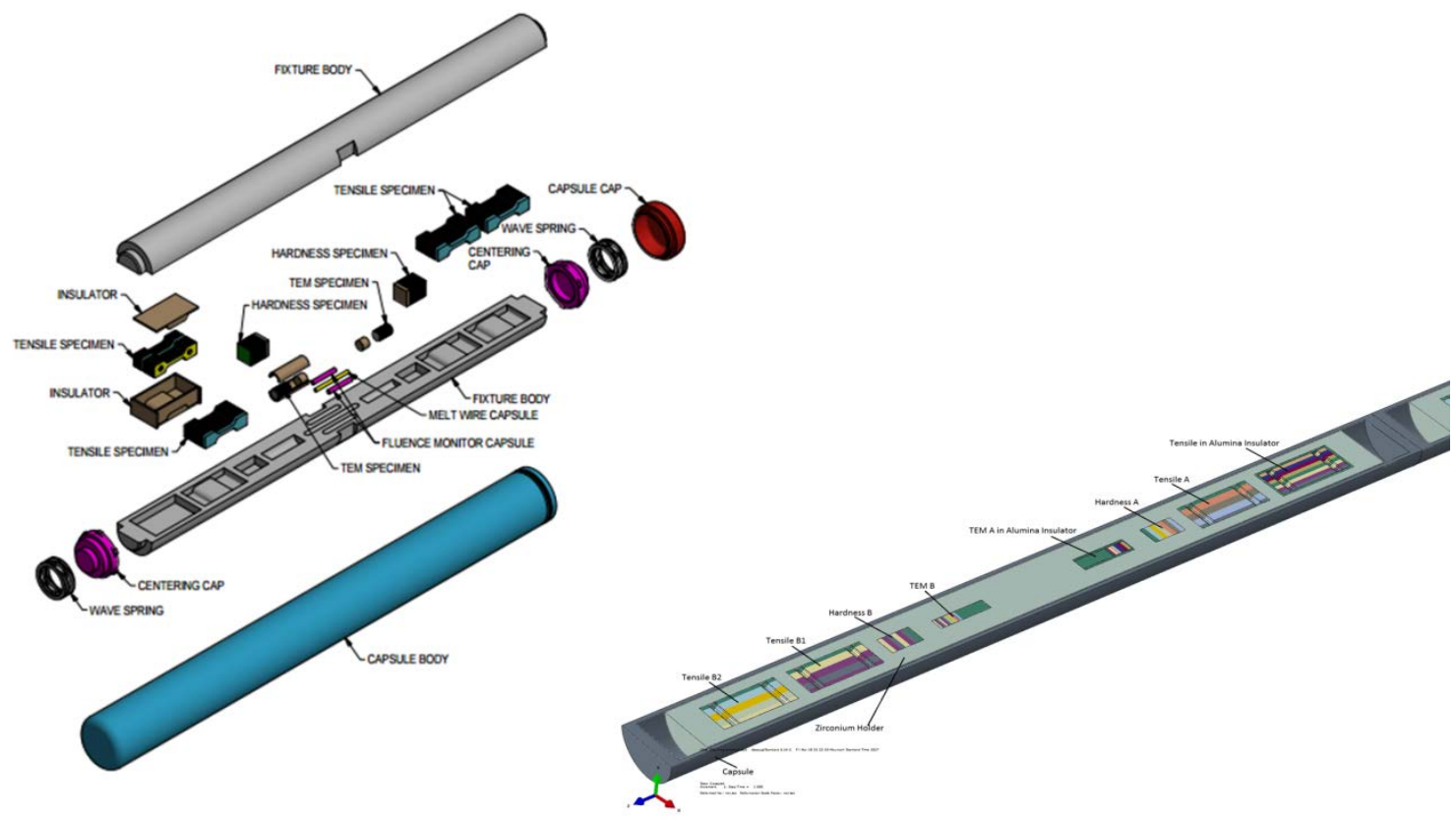

Figure 9. N-SERT Capsule and Fixture Layout

The conceptual design review was completed in January 2017 with the final design review completing on May 25, 2017, meeting the May 31, 2017 "Final Design Complete" milestone. A fabricator has been identified for the machining of the material specimens and work will begin as soon as the contract is signed. The materials for the experiment hardware have been ordered and are expected to arrive by the end of September, at which time INL will begin the fabrication of the capsules and fixtures. Hardware fabrication is to be finished by $11 / 30 / 17$, followed by experiment assembly.

\section{General Electric-Hitachi 10393}

The objective of the GEH-10393 experiment is to perform full irradiation and PIE on materials produced by direct metal laser melting (DMLM) or additive manufactured (AM) material fabrication. The data 
obtained will be used to assess the changes in irradiated AM material properties as compared to unirradiated material. It is desirable to test material fabricated in this manner because there are significant opportunities for implementation as reactor internal repair parts, fuel debris resistant filters, and fuel spacers in existing light water reactors (LWRs). Significant work has been performed to evaluate and confirm that DMLM materials, commonly referred to as AM materials, are capable of achieving equivalent properties to wrought materials while facilitating the manufacture of parts with unique design features. For the use of these materials in advanced power applications such as LWR or small modular reactor (SMR) internal components subjected to irradiation, industry as a whole needs to evaluate the effects of irradiation on the material properties. These data will help industry to achieve improved designs with additional cost benefits as well.

The GE-Hitachi experiment was initiated in the first quarter of FY17. The experiment is a drop-in static materials experiment and will be irradiated in position B11 at the ATR. The specimens to be irradiated in this experiment will be manufactured and machined by GE-Hitachi. The experiment contains one capsule that will hold 12 round compact tension specimens and eight ASTM standard sub-size tensile specimens (see Figure 10). The experiment assembly was designed to include instrumentation (i.e. melt wires, $\mathrm{SiC}$ temperature monitors and flux wires) that will be analyzed during PIE. The basket is designed to achieve capsule and experiment assembly orientation requirements with respect to the reactor core center-line.

The design was approved in the second quarter and fabrication and irradiation preparation tasks were completed in the third quarter of FY17. The experiment has been inserted into ATR and irradiation will begin upon startup of cycle 162A, currently scheduled for early October, 2017, and will continue irradiation for three cycles, reaching approximately 150 effective full power days.
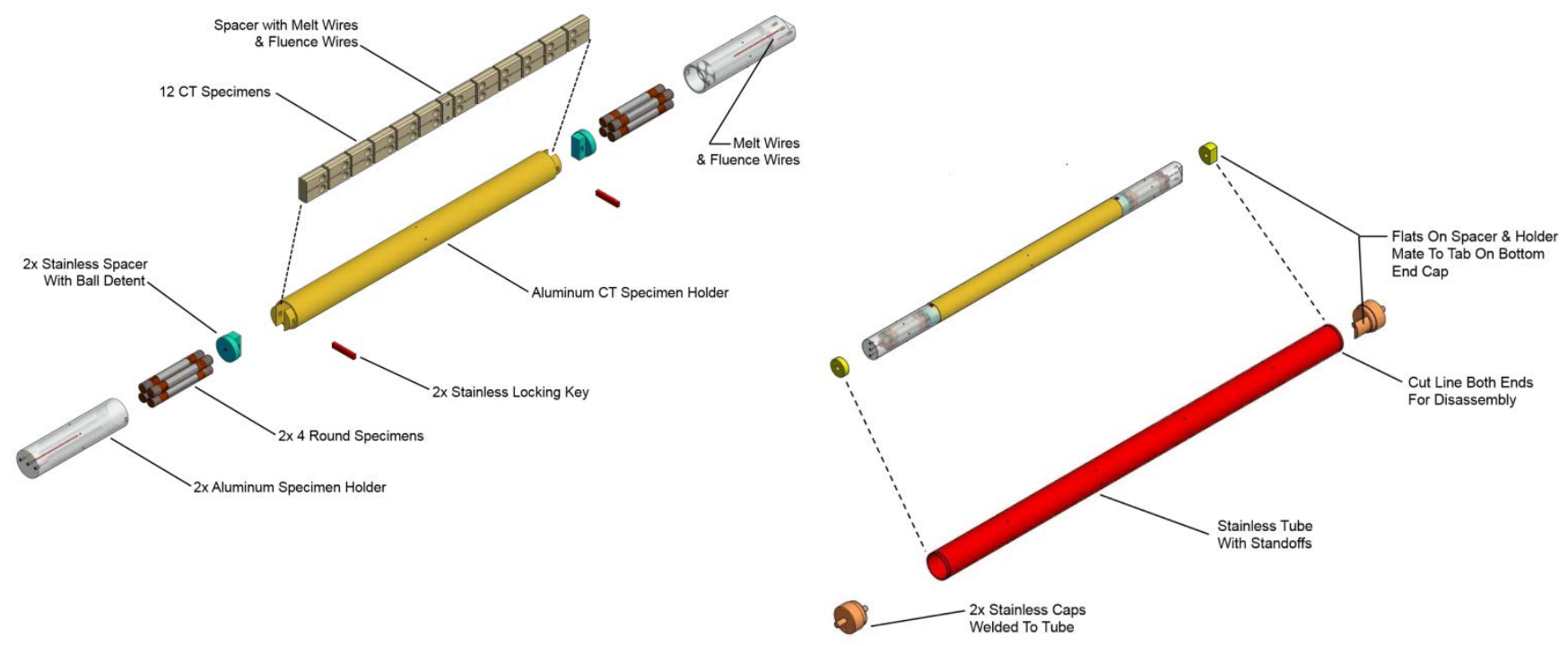

Figure 10. Schematic of GEH-10393 Capsule and Specimens 


\section{SHIPPING}

Technical support on radioactive material shipments was provided to NSUF projects, including package selections, shipping cost estimates, and coordination and scheduling between multiple facilities. Procurement of three new type A shipping containers was completed early in the fiscal year and containers were delivery to INL in March 2017. On June 21, 2017, the milestone to receive irradiated University of California, Berkeley (UCB) specimens from Pacific Northwest National Laboratory (PNNL) was met and the SAM-1 experiment was successfully shipped, processed at International Isotopes, Inc. and then the specimens were returned to INL in December 2016 and January 2017. In addition, receipt of Ultra and Drexel MAX specimens from MIT Reactor were received at the INL's Materials and Fuels Complex in September, meeting a project level 3 milestone.

These shipments add to the quantity and diversity (i.e., uniqueness) of materials that are available in the library and expands research opportunities for scientists and researchers around the US and the international materials community. An important objective is to have a consolidated centralized materials library and these shipments complete that objective.
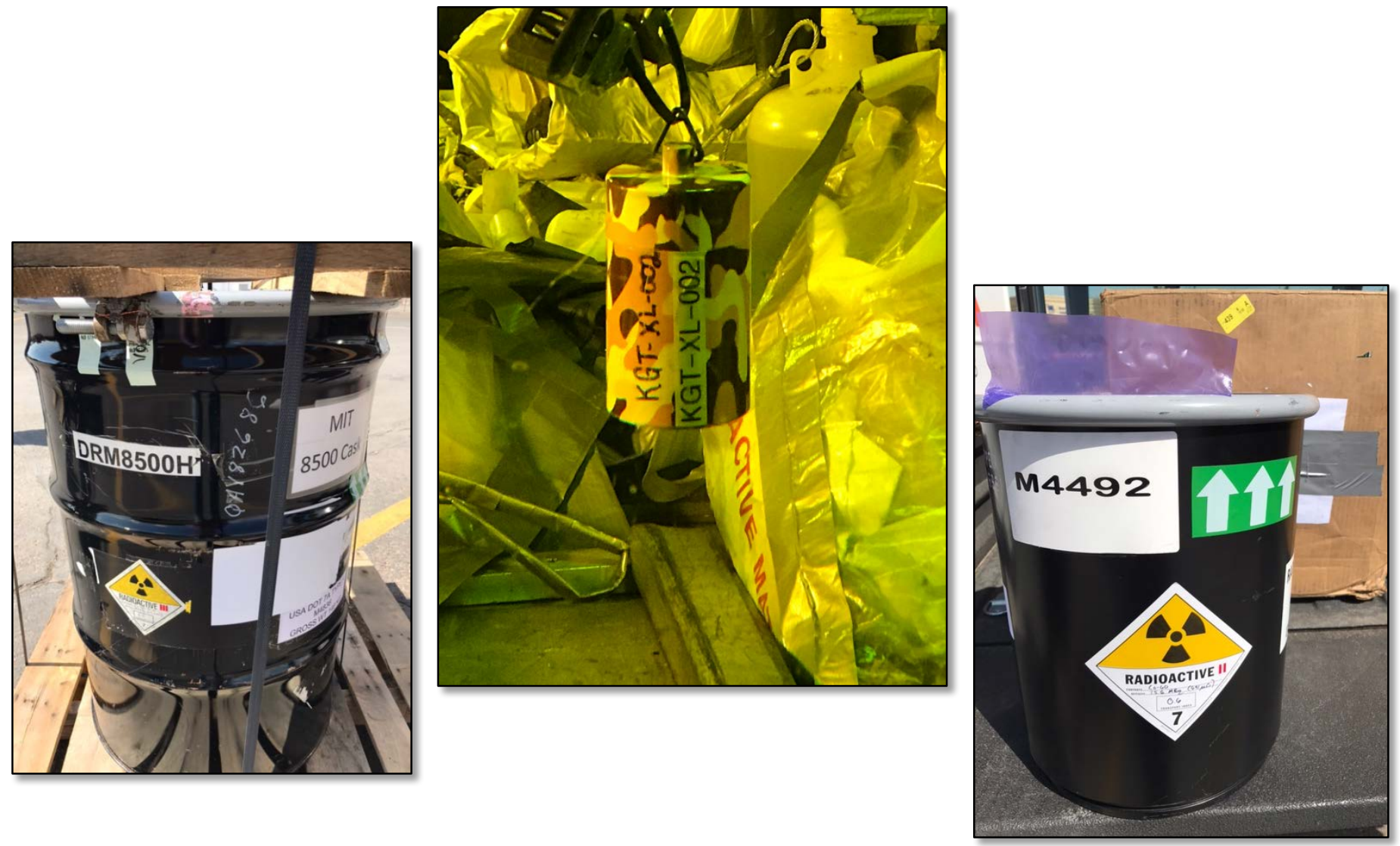


\section{MILESTONE SUMMARY}

\begin{tabular}{|c|c|c|}
\hline Milestone \# & Description & $\begin{array}{l}\text { Completion } \\
\text { Date }\end{array}$ \\
\hline M3UF-17IN0207064 & CSM-10584 Design Review Complete & $6 / 30 / 17$ \\
\hline M3UF-17IN0207133 & ISU-10537 Design Review Complete & $5 / 25 / 17$ \\
\hline M3UF-17IN0202055 & $\begin{array}{l}\text { Complete shipment of Ultra and MAX specimens from MITR to } \\
\text { INL }\end{array}$ & $9 / 13 / 17$ \\
\hline M3UF-17IN0202057 & $\begin{array}{l}\text { Complete Shipment of UCB Experiment contents from PNNL to } \\
\text { INL }\end{array}$ & $6 / 21 / 17$ \\
\hline M3UF-17IN0202063 & $\begin{array}{l}\text { Complete preparation for insertion of UCF-3 into ATR (Ready-to- } \\
\text { insert) }\end{array}$ & $11 / 10 / 2016$ \\
\hline M3UF-17IN0202062 & Complete Final Design of the BSU Experiment & $12 / 20 / 2016$ \\
\hline M3UF-17IN0207166 & $\begin{array}{l}\text { GEH } 10393 \text { Complete preparations for insertion into ATR (ready } \\
\text { to insert) }\end{array}$ & $5 / 23 / 2017$ \\
\hline M3UF-17IN0202058 & $\begin{array}{l}\text { Submit Year End Experiment Planning and Management } \\
\text { Summary Report }\end{array}$ & $9 / 22 / 2017$ \\
\hline
\end{tabular}

Table 1. Milestone Summary 
Appendix A

Process Flow

\section{Process Flow}

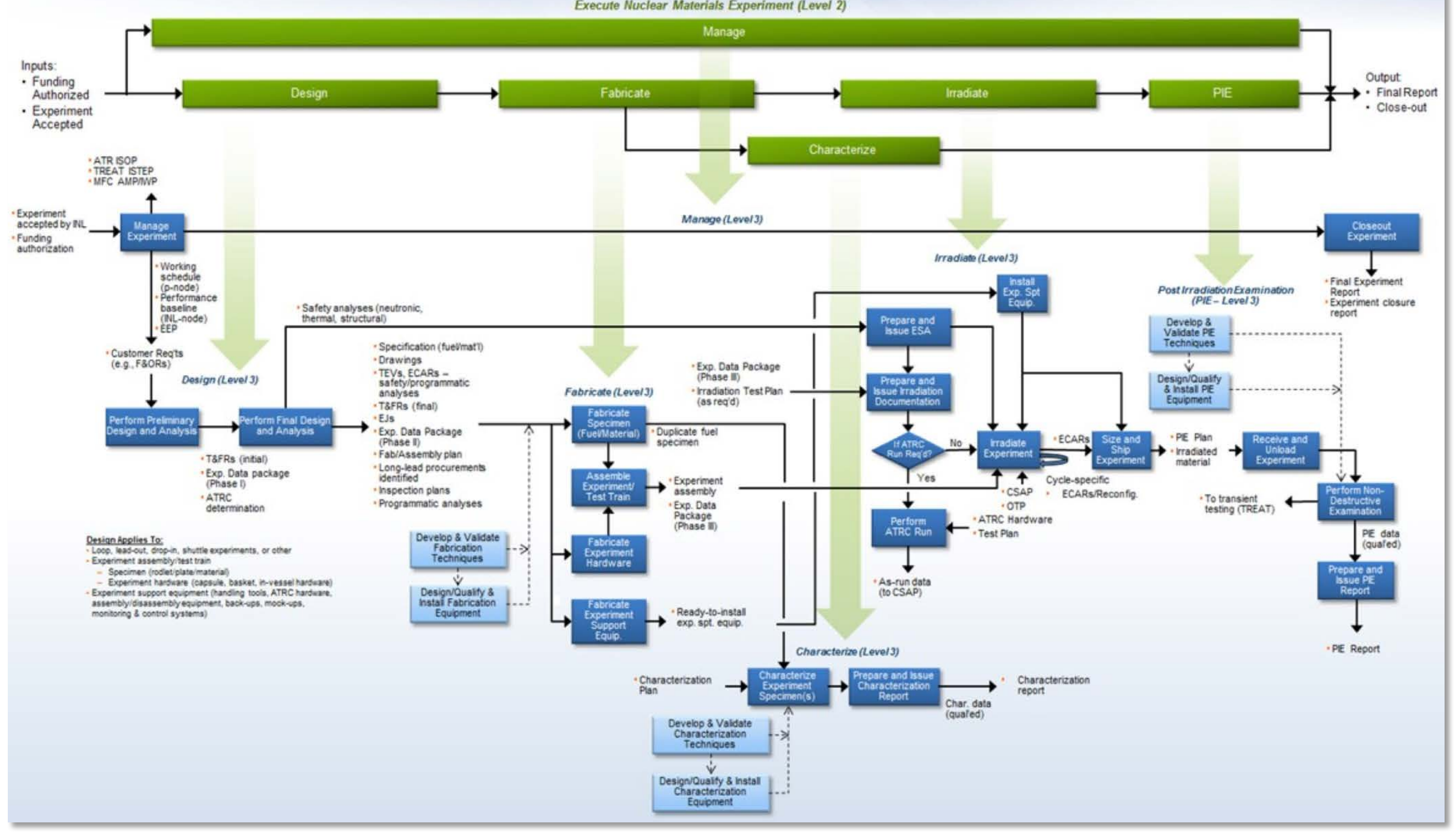

